\title{
An optimized hadrontherapy installation based on multiple-extraction, ultra-fast cycling FFAG
}

\author{
F. Meot, Y. Mori, E. Brebant, G. Cappai, \\ J. Collot, H. Mammar, C. Mazzara, F. Tricart
}

\author{
Collider-Accelerator Department \\ Brookhaven National Laboratory \\ Upton, NY 11973
}

Notice: This document has been authorized by employees of Brookhaven Science Associates, LLC under Contract No. DE-AC02-98CH10886 with the U.S. Department of En ergy. The United States Government retains a nonexclusive, paid-up, irrevocable, world-wide license to publish or reproduce the published form of this document, or allow others to do so, for United States Government purposes. 


\section{An optimized hadrontherapy installation based on multiple-extraction, ultra-fast cycling FFAG}

François Méot, Dr (a), Yoshiharu Mori, Pr, Dr (b), Emilie Brébant, Architect (c), Giovanni Cappaï, Architect (c), Johann Collot, Pr, Dr (d), Hamid Mammar, Pr, MD (e), Christophe Mazzara, Dr (f), François Tricart, Engineer (g)

Authors'institutional affiliations :

\section{(a) Corresponding author.}

Brookhaven National Laboratory, C-AD, Upton, NY, 11973-5000, USA

Tel. : +1 631344 8204, mail : fmeot@bnl.gov

(b) Kyoto University Research Reactor Institute, 2 Asashiro-Nishi, Kumatori-cho, Sennan-gun, Osaka 590-0494 JAPAN. Tel. : +81-72-451-2300, FAX : +81-72-451-2600

(c) Agence Architecte Giovani-Cappai, 169 Avenue Charles de Gaulle, 69000 Tassin-la-Demi-Lune, France. Tel. : +33 4783429 38, Fax : +33 478347847

(d) LPSC, CNRS-IN2P3, Universite Joseph Fourier, 53 Avenue des Martyrs, 38000 Grenoble, France. Tel. : +334 7628 4001, Fax : +33476284143

(e) Centre Antoine Lacassagne \& Université Sophia Antipolis, 227 Avenue de la Lanterne, 06200 Nice , France. Tel. : +33 4920310 80, Fax : +33492031096.

(f) Institut National des Sciences et Techniques Nucleaires, CEA Saclay, 91191 Gif-sur-Yvette, France. Tel. : +33169083410

(g) SIRR , INGENIERIE SAS, SNC-Lavalin Santé, Le Quatuor - Bâtiment D, 16 Avenue Tony Garnier, 69365 Lyon cedex 7, France. Tel. : +33 3884088 00, Fax : +33 388672280 


\section{Conflict of Interest Notification.}

This R\&D work has been performed by the authors and by them only. It is an original work, based on public and common knowledge.

There exists no actual or potential conflicts of interest of any sort concerning this work, with noone, neither any Institution of any sort.

We are not using copyrighted information, neither are using patient photos. 


\begin{abstract}
Purpose:

Propose an innovative concept of simultaneous beam delivery to several hadrontherapy treatment rooms, based on a technology of multiple-extraction fixed-field synchrotron accelerator, and on adapted hospital architecture. This concept will reduce treatment cost, bring it closer to conventional X-ray cost, making hadronthrapy accessible to greater number of patients.
\end{abstract}

\title{
Materials and Methods
}

Based on fixed-field alternating-gradient accelerators (FFAG) specificities, a hadrontherapy center exploiting multiple extraction, can be devised. The accelerator is located underneath the treatment rooms, themselves arranged in a cylindrical-symmetry layout. Patient preparation and medical areas are fitted into the upper floor, in the central area of the building and between the treatment rooms.

In any treatment room, beam is delivered independently of, and simultaneously with, the other rooms, at typically $100 \mathrm{~Hz}$ rate, ensuring $5 \mathrm{~Gy} /$ minute delivery.

\section{Results}

The FFAG-based hospital building is estimated to 20 MEuro (20\% lower than a comparative, conventional rectangular layout), for $6500 \mathrm{~m}^{2}$ overall surface and assuming 5 bunkers, including 4 gantries and a fixed-beam room. With 14 hours dayly for treatment and allowing a conventional 22 minutes per fraction, treatment capacity is $36 \%$ higher than in a classical 5-room proton installation based on a single extraction beam line and on sequential beam delivery. Compared to the latter, the present innovative structure allows three times the number of patients per full time employee. A medico-economical study and comparison with published state-of-art hadrontherapy center costings 
was conducted and yielded decrease of treatment cost by close to a factor 2 .

\section{Conclusion}

The innovative concept proposed reduces investments, operational costs and treatment cost, hence enhancing economical effectiveness of the hadrontherapy method, thus allowing larger number of patient treatment per year, and fostering the development and affordability of hadrontherapy.

Key words: FFAG, spiral scaling FFAG, fixed field accelerator, medical accelerator, hadrontherapy, dose escalation. 


\section{Introduction}

Hadrontherapy nowadays is based on the use of synchrotron or cyclotron accelerators. However, potential and interest of fixed-field alternating gradient accelerators (FFAG) in this application, in many aspects of which various will be recalled here, have been demonstrated in dedicated studies [1,2]. The FFAG method [3] in particular allows simultaneous extraction with high-repetition rate from multiple extraction ports, using appropriate RF systems [4,5].

Based on the specificities of the FFAG technology, the principles of a hadrontherapy treatment center that would exploit multiple, simultaneous extraction, can be devised [6]. The present report addresses and briefly discusses the various aspects and the potential advantages of such layout, compared to typical most recent hadrontherapy installations.

In particular, Section 2 discusses the present state-of-the-art in the domain of the dedicated accelerator, Section 3 describes the multiple-extraction FFAG capable of high repetition rate, simultaneous beam delivery in a number of treatment rooms, Section 4 presents an optimized scheme of a treatment center based on the cylindrically symmetric arrangement of the accelerator installation, Section 5 discusses the trends that these two innovative concepts (namely, a parallel multi-port beam delivery accelerator and the cylindrically symmetric architecture in which it fits) bring in terms of economical impact on the cost of a treatment session.

This short report does not intend to be an fully achieved demonstration, it is rather intended as a guide through the concepts so introduced and in the discussion of their possible medico-economical impact. 
Nevertheless, much details regarding the accelerator technology can be found on the site of the RACCAM project [7] (a project funded by the French National Research Agency). Documents regarding the detailed costing study can also be found on that very site. Note as well that, the principles developed here concern a proton based installation, yet they can be extended to a compact Carbon FFAG ring with like properties. A proton installation could in addition be seen as a first stage in developing a ion treatment center, where the proton ring could serve as an injector in the Carbon ring.

On the other hand, the interested reader may also refer to detailed comparisons between the existing technologies : cyclotron, synchrotron, linear accelerator, FFAG, including costing consideration, as these have been subject to extensive discussions during the FFAG2010 workshop [7, “Documents Techniques" link]. A detailed costing of the particular FFAG installation addressed in this report can be found on RACCAM web site [7, "Documents Techniques"]. Let us add also that it is not the goal of this paper to discuss other aspects and possible impact on installation costs as beam lines, beam manipulation methods and other gantries, the principles of multi-port simultaneous beam delivery as introduced here being to a large extent decoupled from these preoccupations (indeed, as a matter of fact, in the principle scheme of an hospital layout introduced below, impact on costing assumes 4 regular gantry systems in 4 rooms on a total of 5).

\section{State-of-the-art}

The use of hadron beams for radiotherapy has been proposed in 1946 [8]. The technique leans on the property of the "Bragg peak" which allows 3-dimensional conformal irradiation and depth tracking of motion, whereas the dimensioning of the accelerator (its energy) and of its housing installation are 
directly related to the Bragg peak depth sought.

A scheme of a typical state-of-the-art protontherapy installation is displayed in Fig. 1. The accelerator (a pulsed synchrotron on this scheme, but more often a cyclotron), features a single extraction beam line, which splits towards several treatment rooms further down the line. More than a single extraction port from synchrotrons or cyclotrons is hardly feasible, due to the technological difficulty on the one hand, and due on the other hand to the lack of interest given the limited average intensity delivered by synchrotrons and to the high extraction losses of cyclotrons. Cyclotrons in addition would most ineffectively require a radiation prone energy selection system in a concrete bunker at each extraction line.

The following describes how the FFAG method allows overcoming various critical limitations of the synchrotron and cyclotron technologies in the hadrontherapy application, and devising an effective treatment area layout.

\section{A hadrontherapy installation based on the FFAG method}

The implementation of the principles introduced here is based on scaling FFAG methods as produced by former RACCAM [1,8] and KEK [9] studies, however they can be extended to non-scaling FFAG designs as presently studied elsewhere [10]. Fixed-field accelerator lattice design methods and magnet technologies will not be discussed here, however details can be found in dedicated studies [11,12].

Various techniques for cycle-to-cycle energy variation, a strong argument in the medical application of 
the FFAG technology, have also been studied elsewhere [1,9] and will only shortly be addressed, where relevant, here.

An FFAG ring can be equipped with N extraction systems, distributed around the ring, Fig. 2. Given an acceleration cycle with repetition rate $\mathrm{rr}$, it results that the $\mathrm{N}$ beam lines (and treatment rooms) can deliver beam in a quasi simultaneously way from user's viewpoint, at an average rate for instance of $\mathrm{rr} / \mathrm{N}$, in a practical manner in the $100 \mathrm{~Hz}$ range (synchro-cyclotrons commonly work in the several 100 Hz range), thus allowing 2 Gy par minute dose delivery (nowadays standard) and more, depending on the intensity provided by the FFAG injector system [1], would dose escalation be required in the future.

In order to achieve the high-repetition rate necessary, the FFAG ring is equipped with a sufficiently powerful radio-frequency (RF) acceleration system. Such RF systems with large radial extent and highgradient magnet-core technology have been designed for and experimented earlier on a $150 \mathrm{MeV}$ proton FFAGs [9]. An N-extraction source with about $100 \mathrm{~Hz}$ repetition rate per port would require several accelerating gaps, distributed around the ring.

Fast, single turn extraction can be used, allowing bunch-to-pixel type of scanning, following modern developing standards. Kicker-septum extraction assembly has been experimented [9] and has proven to be appropriate in medical application, whereas specific design studies have demonstrated the technological feasibility using spiral FFAG lattice [1]. Multiple-kicker extraction would further bring the capability of variable energy extraction (amongst other methods of cycle-to-cycle variable energy working regimes, see Ref. [1]) : while the accelerated beam spirals outwards, by synchronizing the extraction kicker on the turn number then extraction energy can be selected. About $50 \mathrm{MeV}$ energy range is feasible. Energy resolution is determined by the acceleration rate, namely a few keV per turn 
for $2 \mathrm{~Gy} /$ minute delivery, which means energy steps with typical $\mathrm{dE} / \mathrm{E}<10^{-3}$, compatible with required resolution on Bragg peak depth in medical applications. Such synchronized double-kicker+septum system has been installed and is routinely used in the EMMA experimental non-scaling FFAG [13].

\section{A dedicated treatment center}

A possible scheme of a dedicated treatment center, taking profit of the $\mathrm{N}$-port simultaneous extraction, is shown in Figs. 3, 4. The cylindrical symmetry of the building is adapted to that of the accelerator system. Each beam line out of the FFAG ring is directed to a treatment room, where beam can be delivered independently of the other rooms at a rate of typically $100 \mathrm{~Hz}$, thus ensuring 2 Gy/minute delivery and beyond.

The FFAG ring is located in the basement of the building, Fig.4. The beam lines have vertical deviation so to reach the horizontal level on the upper floor. Patient preparation rooms and other medical and administrative premises are fitted into this layout, in the central area of the building as well as between the treatment rooms. Potential advantages of the $\mathrm{N}$-extraction polygonal arrangement of the beam extraction and delivery, compared to classical rectangular layout (Fig. 1), are many-fold, as :

- allowing quasi-simultaneous beam delivery in all treatment rooms,

- potentially resulting in more treatments

- and in higher availability of beam time for the patient, as is the trend nowadays (in view of in-situ imaging or beam time consuming gating methods, for instance),

- allowing beam time in dedicated room for R\&D programs as radiobiology, accelerator and beam 
physics, beam manipulation R\&D,

- minimizing the building and architectural surfaces as well as the distances between the various medical zones,

- thus minimizing the distances to be covered by patients and the medical staff,

- minimizing manpower needed to operate the treatment center,

- reducing the building construction and operation costs,

- improving various architectural aspects as lighting, radiation shielding, etc.

These considerations meet the requirement of reducing the treatment cost, so to bring it closer to that of the (X-ray) Intensity Modulated Radio-Therapy (IMRT) and to improve the economical effectiveness of the hadrontherapy method.

\section{Economical impact}

A detailed medico-economical study of an FFAG-based hospital and an estimate of its impact on the cost of a treatment session has been performed recently, based on costings regarding the accelerator, its beam lines and the necessary medical equipments [14], the investment costs in the building and its operation [15], and using a medico-economical model built for that purpose [16]. This study is presently subject to publication as a review article. The main aspects and outcomes are summarized in the following.

Operational cost 
A hadrontherapy center is generally equipped with $\mathrm{N}=2$ to $\mathrm{N}=4$ treatment rooms (called bunkers in the following) which are continuously operated over a given number of hours per day $\left(\mathrm{t}_{\text {day }}\right)$. While the beam is delivered in a bunker, simultaneously in the other treatment rooms time is devoted to patient preparation and after-treatment room reconditionning.

In case the total time for the sequential treatments is longer than the sum of the preparation and reconditioning time, each bunker is affected by a dead-time, quantified by the bunker occupation rate

$\mathrm{BOR}=\left(\mathrm{t}_{\text {prep }}+\mathrm{t}_{\text {treat }}+\mathrm{t}_{\text {rec }}\right) /\left(\mathrm{N} \times \mathrm{t}_{\text {treat }}\right)$

(in the numerical examples here, we consider preparation stage $t_{\text {prep }} \approx 8 \mathrm{~min}$, treatment stage $t_{\text {treat }} \approx 7$ min, patient reconditioning stage $\mathrm{t}_{\mathrm{rec}} \approx 7 \mathrm{~min}$, for a total $\approx 22$ minutes reference session duration). This indicator is $100 \%$ for a classic center with 3 bunkers, and decreases from 4 bunkers on. A bunker working rate "WR" can also be defined, allowing for quality control, maintenance and breakdown periods, etc., and yields the average daily capacity,

$\mathrm{N}_{\text {day }}=\mathrm{N} \times \mathrm{BOR} \times \mathrm{WR} \times \mathrm{t}_{\text {day }} /\left(\mathrm{t}_{\text {prep }}+\mathrm{t}_{\text {treat }}+\mathrm{t}_{\text {rec }}\right)$

Estimates so obtained are represented in Fig. 6 for $t_{\text {day }}=14$ hours daily treatment time. For the considered 22 minutes session time, a sequential organization reaches its maximal capacity with four bunkers and BOR $\approx 92 \%$. Beyond four bunkers the BOR decreases (Fig. 8). The present FFAG-based scheme assumes simultaneous beam delivery to the $\mathrm{N}$ bunkers, allowing $\mathrm{BOR} \approx 100 \%$ whatever $\mathrm{N}$. Treatment capacity is then 36 \% higher, and increases linearly with the number of bunkers. 
A medico-economical model of the operational cost has been built [16] and validated against a series of published models of both IMRT and protontherapy center costings [17-19], yielding a benchmarking mutual agreement within a few \% on several cases of session costing. The estimates addressed below are based on this "home-made” model.

The FFAG-based hospital building is estimated to 20 MEuro, with a surface of $6500 \mathrm{~m}^{2}$. With the most recent parameters [18], and assuming 5 bunkers and a conventional sequential delivery, the cost of a session would be 650 Euro, due essentially to the circular organization of the patient flow (instead of 750 Euro as in a classical proton installation, Fig. 1). However the FFAG-based multi-port parallel beam delivery allows substantial additional optimizations leaning on

- increase of treatment capacity,

- low cost of machine installation and operation,

- smaller surface of the treatment areas compared to nowadays layouts,

- optimized organization of hospital operation close to a X-ray radiotherapy center, namely toward 3050 patients per full-time equivalent personnel (FTE) [17,18,19].

The medico-economical study performed indicates that he reduction in cost ranges from $36 \%$ for 30 patients/FTE to $48 \%$ for an IMRT-like 56 patients/FTE ratio, which could be regarded as an achievable goal given the multi-port, parallel beam delivery.

These costs are given as indicative figures, and should not be taken in an absolute manner. What has to be considered rather, is the relative decrease in cost introduced by the concepts introduced here.

\section{Conclusion}


The multi-port, parallel beam delivery FFAG ring technique on the one hand, and the treatment area layout described on the other hand, can be applied, mutatis mutandis, to a carbon installation. In particular an accelerator providing about $30 \mathrm{~cm}$ Bragg peak depth (about $430 \mathrm{MeV} / \mathrm{u}$ ), together with its FFAG injector (about $60 \mathrm{MeV} / \mathrm{u}$ carbon, $230 \mathrm{MeV}$ proton) would fit into the ground floor room as schemed in Figs. 3, 4.

\section{Acknowledgements}

We would like to thank Samuel Meyroneinc, from the Institut Curie, Centre de Protontherapie d’Orsay, for his much appreciated help in discusing the costing study.

\section{References}

[1] S. Antoine, B. Autin, W. Beeckman, et al., Principle design of a protontherapy, rapid-cycling, variable energy spiral FFAG, NIM A 602 (2009) 293-305.

[2] T. Yokoi, FFAG Accelerator for Ibaraki Proton Therapy Facility, FFAG 2005 Workshop, KURRInstitute, Kyoto (2005). http://hadron.kek.jp/FFAG/

[3] Ch. Prior, FFAG Accelerators, in Beam Dynamics Newsletter No. 43, Aug. 2007, pp. 14-143. 
[4] C. Ohmori, F. Méot, J, Pasternak, High-field gradient RF system for a spiral FFAG, RACCAM, Procs. EPAC 08 Conference, Genoa. http://www.jacow.org/

[5] A. Takagi, Y. Mori, J. Nakano, et al., Radio frequency acceleration system for $150 \mathrm{MeV}$ FFAG, Procs. 2003 Particle Accelerator Conference, Portland, Oregon. http://www.jacow.org/

[6] F. Méot, Principle of an innovative hadrontherapy installation, based on the RACCAM fixed-field synchrotron, internal report CNRS/IN2P3 LPSC-10-21 (2010). http://psc.in2p3.fr/service accelerateurs/raccam.htm

[7] RACCAM web site : http://lpsc.in2p3.fr/service accelerateurs/raccam.htm

[8] R. R. Wilson, Radiological use of fast protons, Radiobiology 47 (1946) 487-491.

[9] S. Machida, Status of 150 MeV FFAG synchrotron, Procs. 2003 Particle Accelerator Conference, Portland. http://www.jacow.org/

[10] T. Yokoi, Status of PAMELA, an overview of UK particle therapy facility using NS-FFAG, Hadrontherapy Workshop, Erice, 29 Apr. 2009. http:/erice2009.na.infn.it/programme.htm

[11] T. Planche, J. Fourrier, J.L. Lancelot, et al., Design of a prototype gap shaping spiral dipole for a variable energy protontherapy FFAG, NIM A 604 (2009) 293-305.

[12] T. Adachi, A 150 MeV FFAG synchrotron with “return-yoke free” magnet, Procs. 2001 Particle 
Accelerator Conference, Chicago. http://www.jacow.org/

[13] R. Barlow ${ }^{\mathrm{f}, \mathrm{h}}$, J. S. Berg ${ }^{\mathrm{b}}$, N. Bliss, et al., Acceleration in the linear non-scaling fixed field alternating gradient accelerator EMMA, to be submitted for publication in Nature (2011). EMMA web site : http://www.conform.ac.uk/documents/emma/

[14] F. Méot , Cout de l’installation RACCAM, Int. rep. CNRS/IN2P3 LPSC (RACCAM) (2009), http://lpsc.in2p3.fr/service accelerateurs/raccam.htm.

[15] Agence Architecte Giovani-Cappai, 169 Avenue Charles de Gaulle, 69000 Tassin-la-Demi-Lune (2009-2010).

[16] C. Mazzara, Etude medico-economique d'un centre de protontherapie equipe d'un accelerateur FFAG a extraction multiple : RACCAM, Internship report, CNRS/IN2P3 /LPSC (2010), http://lpsc.in2p3.fr/service accelerateurs/raccam.htm.

[17] M. Huybrechts, C. Obyn, J. Gailly, et al., Health Technology Assessment (HTA), Brussels: Federaal Keniscentrum voor de Gezondheidszorg (KCE). KCE reports 67A. 2007.

[18] A. Peeters, J.P. Grutters, M. Pijls-Johannesma, et al., How costly is particle therapy? Cost analysis of external beam radiotherapy with carbon-ions, protons and photons. Radiother Oncol. 2010 Apr; 95(1):45-53

[19] P. Lambin, H. Fekkers, E. Daniels, et al., Euregional ion therapy institute. Maastricht: Business 
Plan; Internal report, unpublished. 2005.

\section{Figure captions}

Fig.1. Typical layout of a state-of-art hadrontherapy installation - here Loma Linda, USA, proton beams. Typical size of the building $45 \times 85$ meters.

Fig. 2. An FFAG ring with five extraction ports, liable to deliver proton beams to five independent treatment rooms simultaneously. Diameter of the ring is 8 meters.

Fig. 3. A top view of a hadrontherapy treatment center with five rooms, exploiting a $2 \pi / N$ symmetry which the FFAG method allows. The FFAG ring is located in the basement of the building, visible in the center part in the layout.

Fig. 4. A cross-sectional view of the polygonal layout hadrontherapy installation, with the FFAG ring on the underground floor, and treatment and medical areas on the ground floor. The footprints of both a proton FFAG (8 meter diameter) and a Carbon FFAG (15 meter diameter) are shown in the central part.

Fig. 5. Planning of treatment sessions based on 4 rooms ("salle 1,..., salle 4" on the figure) in a sequential (top) and in a parallel (bottom) beam delivery scheme.

Fig. 6. Treatment capacity (sessions per day) in a classical sequential beam delivery for reference ("REF" curve), and in three different operational modes of the FFAG-based installation : fully 
sequential with a regular 17 patients/FTE (1), fully parallel with an intermadiate 30 patients/FTE (2), fully parallel with 56 patients/FTE as achieved in X-Ray therapy centers (3). 


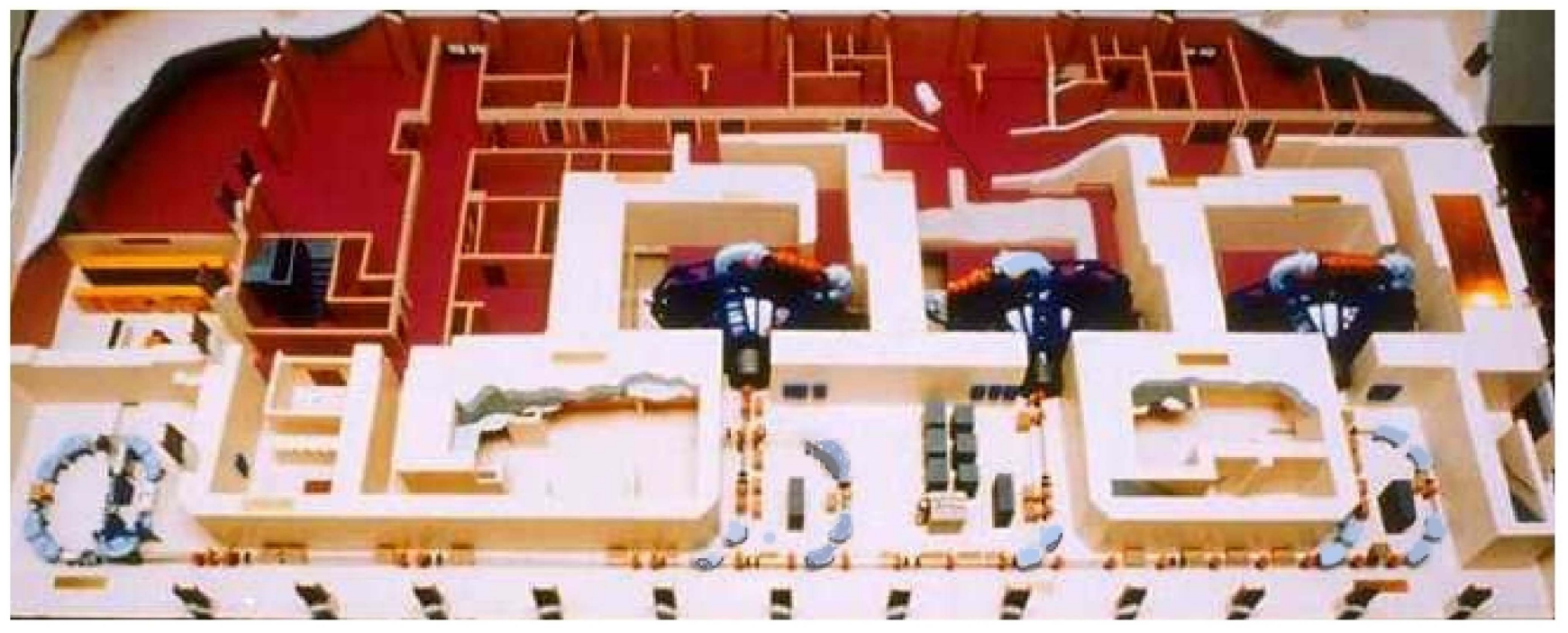

Figure 1

Figure 2

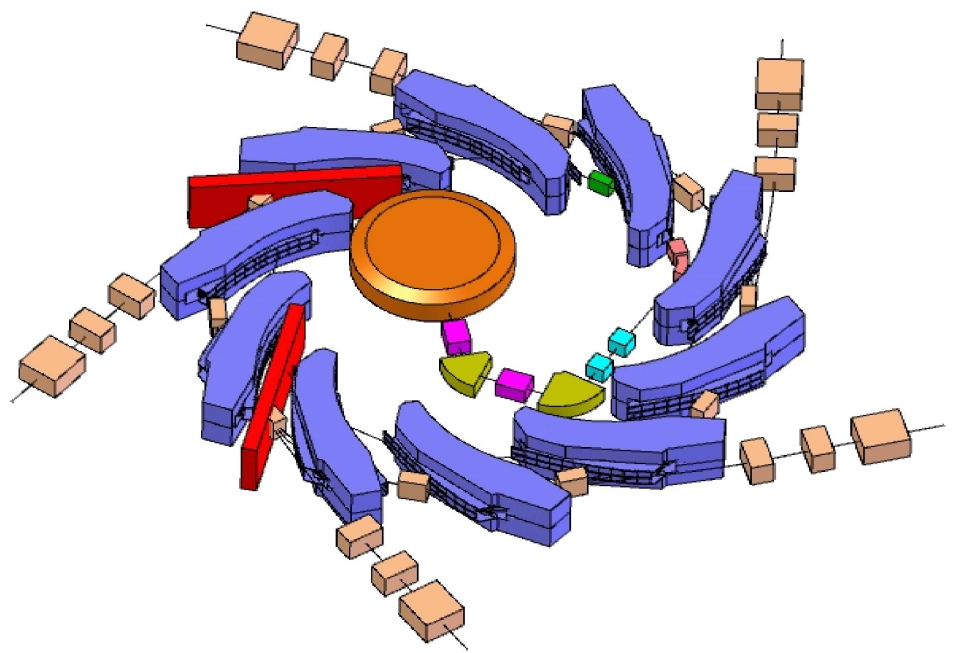




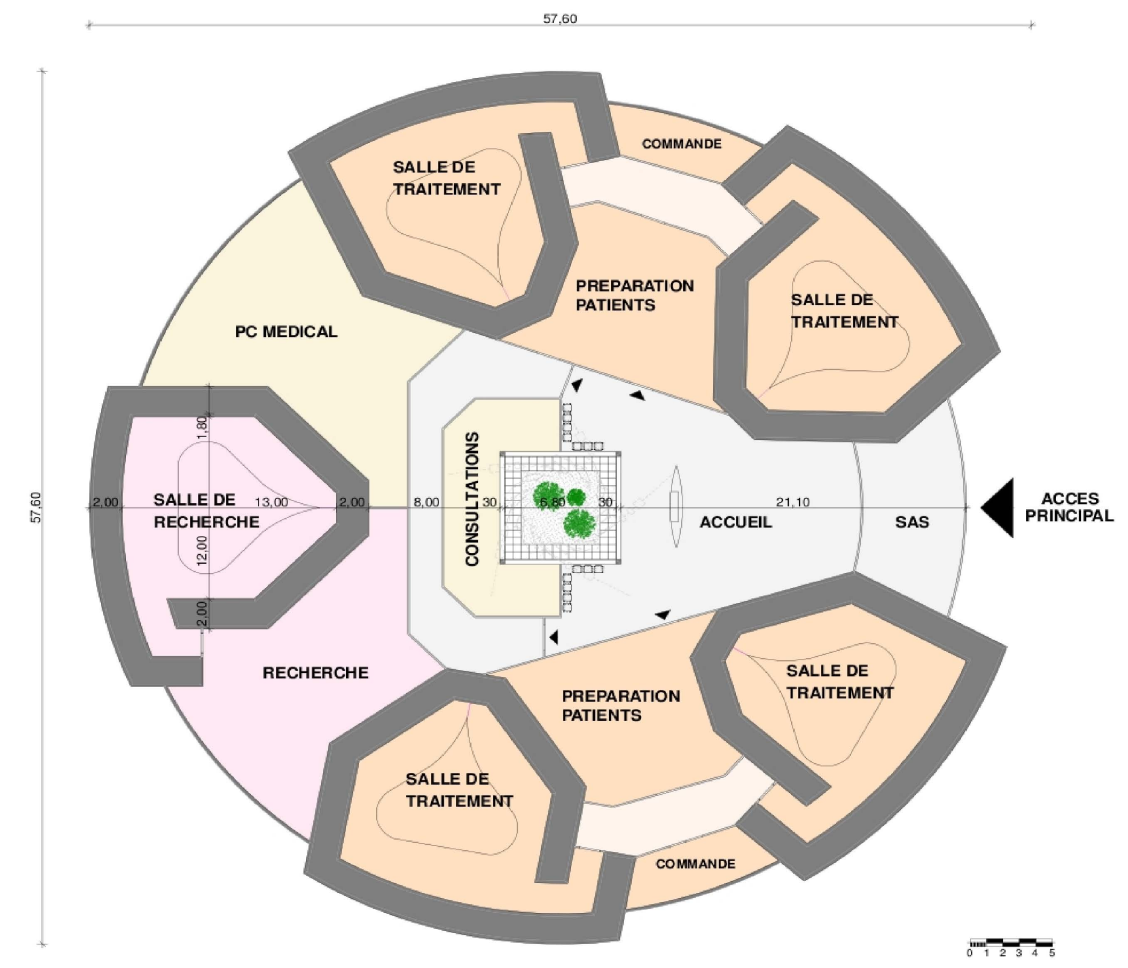

Figure 3

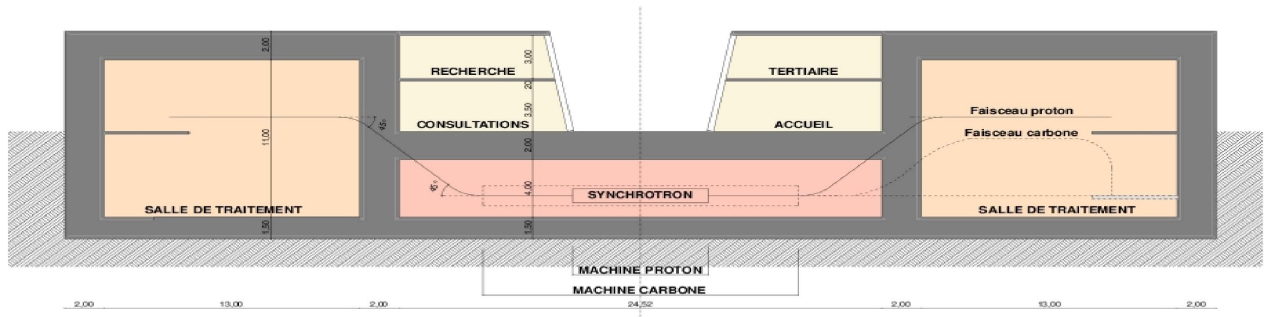

Figure 4 
s alle 1 5 alle 2 $s$ alle 3

$$
\text { s alle } 4
$$

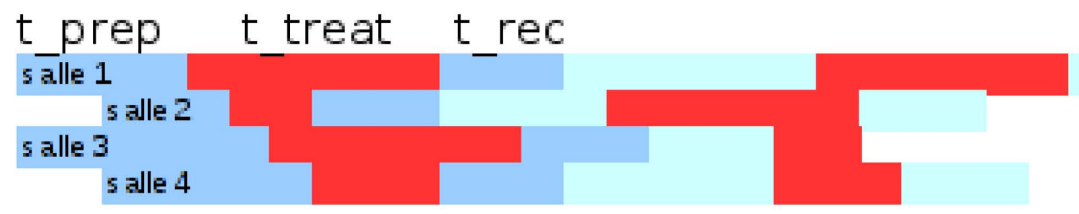

Figure 5

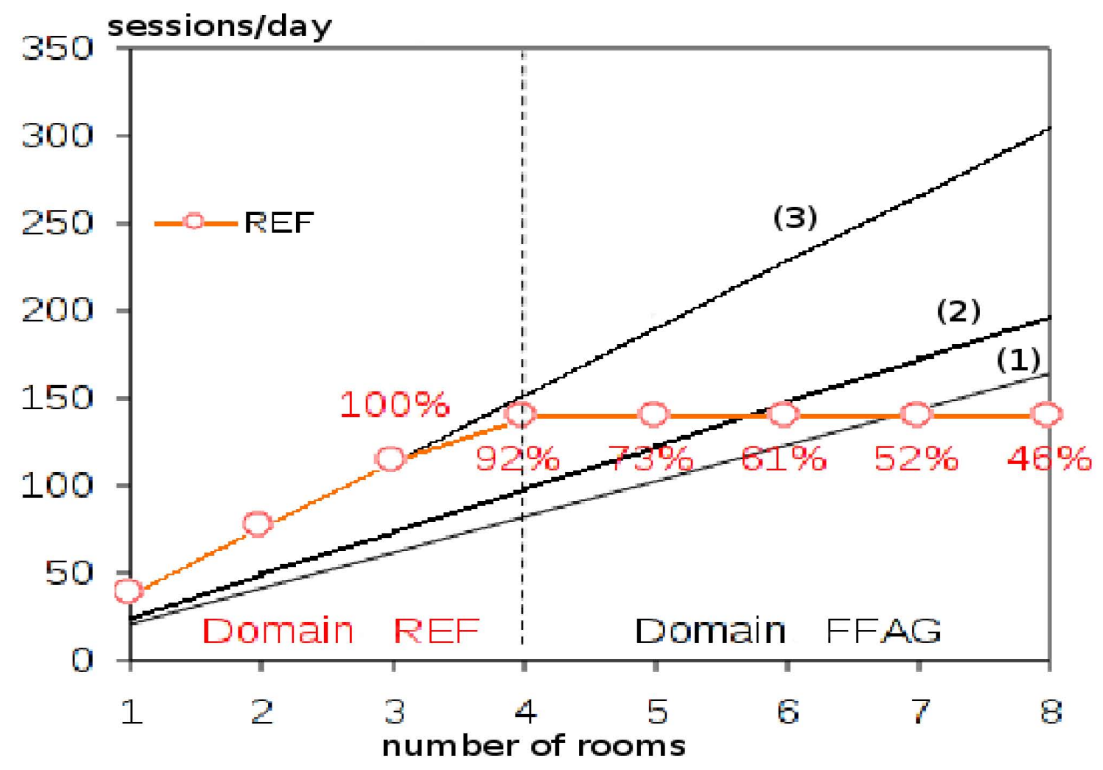

Figure 6 